\title{
A Motion Control Algorithm for the Robot Expression of the Emotion Extracted from SMS Text
}

\author{
Dong Yup Choi ${ }^{1}$, Jinkyu Park ${ }^{2}$ \\ ${ }^{1}$ Daelim University College, Department of Mechatronics. \\ 20 Imgokro Dongahngu Anyang, Gyeonggido, Republic of Korea \\ dychoi@daelim.ac.kr \\ ${ }^{2}$ ERAP Korea Co. LTD \\ 16 Hansan-gil Cheongbuk Pyeongtaek,Gyeonggi-do Republic of Korea \\ retri@4drobot.com
}

\section{Extended Abstract}

Recently, many studies have been made on the emotion expression robot. Emotion of robot is usually expressed by facial expression, gesture, and voice which are generally used by human to express emotion. In the case of [1], facial expression, gesture and voice were used for the expression of emotion, and in the case of [2][3] and [4], facial expression was used.

In order to express an emotion for the robot, firstly the kind of the emotion to be expressed must be recognised by the robot. And the emotion is expressed by predetermined method by facial expression, voice, gesture and so on. In the case of [1], the emotion to be expressed was extracted from SMS text using neural network and hidden markov model as [5] .

The robot in [1] makes a gesture which was predetermined according to the emotion each time a kind of emotion is extracted from the SMS text. A series of motion is needed to express an emotion. And for the smooth motion, many points must be used for one emotion expression. Therefore, it is not easy to make enough number of predetermined gestures to express all kinds of emotion.

The purpose of this study was to develop the algorithm to create a new predetermined gesture easily. For the purpose of easy creation of predetermined gesture, key frame concept was adopted. Key frame is a robot pose that is critical in order to express the emotion. A predetermined gesture is composed of several key frame. And one or more key frame can be added or removed easily to modify the gesture.

For the smooth motion between adjacent two key frames, the portion between adjacent two key frames is divided into a few parts and PWM algorithm is applied to each part.

For the creation and modification of a predetermined gesture, programming tool was developed. In this programming tool, a gesture can be created and modified by editing the number of key frames and the angle of each joint. With this programming tool, not only robot engineer but also anyone, who is not expert in robotics, can easily create or modify a predetermined gesture.

In this study, a motion control algorithm was developed for the robot expression of the emotion extracted from SMS text. Key frame concept was adopted for the easy creation and modification of an emotional gesture. Programming tool was also developed for the editing of the key frame sequence and joint angle.

This work was supported by the National Research Foundation of Korea grant funded by the Korean government as a General research support project (NRF-2011- 0025478).

\section{References}

[1] D. Choi and J. Park, "Simulation Robot for the Expression of Emotion Extracted from SMS message," in Proc. of $3^{\text {rd }}$ international Conference on Mechanical Eng. And Mechatrnics, Prague, Czech Republic, 2014, pp 71-1 - 71-4.

[2] J. Park, W. Kim, W. Lee, and M. Chung, "Life-like facial expression of mascot-type robot based on emotional boundaries," Journal of Korea Robotics Society, vol. 4, no. 1, pp 281-288, 2009. 
[3] S. Sosnowski, A. Bitterman, K. Kuhnlenz, and M. Buss, "Design and Evaluation of Emotion-Display EDDIE," in Proc. of the 2006 IEEE/RSJ, International Conference on Intelligent Robots and Systems, Beijing, China, 2006, pp. 31133118.

[4] T. Usui, K. Kume, M. Yamano, and M. Hashimoto, "A Robotic KANSEI Communication System Based on Emotional," in Proc. of the 2008 IEEE/RSJ, International Conference on Intelligent Robots and Systems, Nice, France, 2008, pp. 3344-3349.

[5] D. Choi, J. Park, and T. Kim, "An Emotion Extraction Method from SMS Text for the Emotion Expression Robot," in Proceedings of the Asia Pacific Conference on Engineering and Applied Science NS_PART3, Osaka Japan, 2015, pp.6366. 